\title{
FIVE-DIMENSIONAL QUASISPIN AND THE SPECTRA OF ODD-PARITY YRAST CONFIGURATIONS IN THE Ba REGION ${ }^{\dagger}$
}

\author{
K.J. WEEKS and J.P. DRAAYER
}

Department of Physics and Astronomy, Louisiana State University, Baton Rouge, Louisiana 70803, USA

and

K. T. HECHT

Department of Physics, University of Michigan, Ann Arbor, Michigan 48109, USA

Received 8 August 1983

\begin{abstract}
The 5-dimensional quasispin formalism is used to evaluate the $(n, T)$-dependent factors of the matrix elements of abnormal parity operators of relevance for yrast-band spectroscopy. The formalism allows the leading pseudo-SU(3) representation of the normal (N) parity part of a shell-model configuration to be coupled to low-seniority states of the abnormal (A) parity high- $j$ intruder part of the configuration. To illustrate the importance of the $n_{\mathrm{A}}, T_{\mathrm{A}}$ dependence of the (A) to $(\mathrm{N})$ space-coupling matrix elements this model is applied to the negative-parity yrast spectra of ${ }^{127} \mathrm{La}$ and ${ }^{127} \mathrm{Ba}$, using hamiltonian parameters which give a reasonable fit for ${ }^{126} \mathrm{Ba}$. The ${ }^{127} \mathrm{La}$ spectrum is reproduced with a seniority-one truncation of the $h_{11 / 2}$ configuration; the ${ }^{127} \mathrm{Ba}$ spectrum indicates a need for higher-seniority admixtures.
\end{abstract}

\section{Introduction}

Until recently microscopic shell-model studies of defonmed nuclei have been restricted to the very lightest deformed nuclei (e.g. in the first half of the 2s1d shell). In heavier deformed nuclei the enormous dimensionalities of a conventional shell-model treatment have precluded shell-model calculations for such systems. Recently, however, it has been shown that group-theoretical methods may be used to reduce the shell-model spaces for strongly deformed heavy nuclei to physical subspaces of tractable size ${ }^{1}$ ). These permit a microscopic study of yrast-band phenomena despite the necessarily severe basis truncation ${ }^{2}$ ). In heavy nuclei the single-particle shell-model levels are characterized by the appearance in each major oscillator shell of a single high- $j$ intruder level of opposite or abnormal parity, e.g. the $h_{11 / 2}$ level which is pushed into the midst of the $g_{7 / 2} \mathrm{~d}_{5 / 2} \mathrm{~d}_{3 / 2} \mathrm{~s}_{1 / 2}$ levels by the strong spin-orbit interaction. An important part of the basis specification involves the partitioning of the valence nucleons into two sets, those which occupy the natural (normal, N) parity sublevels of a major oscillator shell and those which occupy the unique (abnormal, A) parity high- $j$ intruder level. Although the possibility of pair promotion from $\mathrm{N}$ - to $\mathrm{A}$ - or $\mathrm{A}$ - to $\mathrm{N}$-orbitals may be included, each piece of the basis is built from weak-coupled state vectors, coupling specific $\mathrm{N}$ - and A-space

+ Supported in part by the US National Science Foundation. 
state vectors. In heavy deformed nuclei a severe basis truncation may be achieved if the $\mathrm{N}$-space vectors are dominated by the leading (most deformed) representations of pseudo-SU(3) symmetry. The pseudo-SU(3) model has been described elsewhere ${ }^{3}$ ). It originated from studies of pseudo spin-orbit doublets ${ }^{4}$ ) and is based on a correspondence such as $\left(\mathrm{g}_{7 / 2} \mathrm{~d}_{5 / 2} \mathrm{~d}_{3 / 2} \mathrm{~s}_{1 / 2}\right) \rightarrow\left(\tilde{\mathrm{f}}_{7 / 2} \tilde{\mathrm{f}}_{5 / 2} \tilde{\mathrm{p}}_{3 / 2} \tilde{\mathrm{p}}_{1 / 2}\right)$ which maps the normal-parity $(\mathrm{N})$ single-particle levels of the $50 \leqslant Z \leqslant 82$ nuclei onto the states of a complete major pseudo-oscillator shell (in this case with major pseudo-oscillator quantum number $\tilde{N}=3$ ). In regions of deformation where single-particle Nilsson orbitals are characterized by good asymptotic pseudo-oscillator quantum numbers $\left[\tilde{N} \tilde{n}_{z} \tilde{\Lambda}\right] \Omega$, the normal-parity configurations are dominated by the leading irreducible representation ${ }^{5}$ ) of pseudo-SU(3) symmetry, $(\lambda \mu)$, with highest possible $2 \lambda+\mu$, and for fixed value of this sum, the highest $\mu$. The goodness of asymptotic pseudooscillator quantum numbers has recently also been exploited in an analysis of the qualitative features of quasiparticle motion in rotating potentials ${ }^{6}$ ).

Although the pseudo-SU(3) approximation for the normal-parity subspace is the key to a tractable basis truncation in heavy deformed nuclei, a further truncation of the abnormal-parity subspace may also be necessary, particularly in nuclei such as those in the Ba region where the high- $j$ intruder level may be being filled by a large number of both neutrons and protons. In a rich valence space of many $j$ sublevels, in a heavy deformed nucleus, seniority is not a good quantum number. However, in a single high $j$-intruder orbit, isolated in a lower major shell, the effects of seniority-breaking forces are inhibited, and the seniority scheme for the single $j$-shell may provide a suitable basis for truncation. The five-dimensional quasispin formalism then serves as a very useful tool in evaluating matrix elements in the high $j$-intruder subspace since it gives the $n, T$ dependence of all matrix elements. The number of nucleons and isospin of the A-subspace may play a vital role in determining the characteristics of the yrast rotational bands in odd nuclei. In ref. ${ }^{7}$ ) it has been shown that it is the A-space $n, T$ dependence of certain crucial coupling matrix elements which determines whether the odd-parity yrast spectrum of an odd-mass nucleus is of stretched ( $\Delta I=2$ sequence) or of ordered ( $\Delta I=1$ rotational sequence) character. Moreover, this $(n, T)$-dependent signature can be used to predict the character of the yrast spectra of odd nuclei throughout the intermediate and heavy mass regions.

Since the five-dimensional quasispin formalism has been an important tool in the microscopic study of yrast-band phenomena in heavy deformed nuclei a review of this formalism as applied to this problem may be useful. The purpose of the present article is twofold. On the one hand, it is our aim to give the detailed $(n, T)$-dependent factors for the A-space matrix elements relevant for yrast-state spectroscopy. For this reason sect. 2 gives a brief review of the five-dimensional quasispin formalism and the quasispin method of calculation. It also gives the general features of the weak-coupling scheme which combine the normal- and abnormal-parity subspaces, in order to identify the quasispin operators whose matrix elements are needed for 
applications of this coupling scheme. A tabulation of the most important matrix elements, in terms of general algebraic $(n, T)$-dependent expressions, is given in an appendix. Our second aim is to illustrate the importance of the $n, T$ dependence of the $\mathrm{A}$ - to $\mathrm{N}$-space coupling matrix elements with a specific example. For this purpose we have chosen the negative-parity yrast spectra of the odd- $A$ neighbors of ${ }^{126} \mathrm{Ba}$. The calculations are presented in sect. 3. A model hamiltonian is chosen which gives a reasonable fit to the yrast states of ${ }^{126} \mathrm{Ba}$. When applied to the negative-parity yrast spectra of ${ }^{127} \mathrm{La}$ and ${ }^{127} \mathrm{Ba}$ this hamiltonian leads to qualitatively different spectra in the odd-proton and odd-neutron nuclei. Although our basis truncation for the odd nuclei may be somewhat too severe for good quantitative agreement with experimental spectra, the qualitative features of the observed spectra are predicted by the model wave functions. These wave functions give us a microscopic basis for the comparison of $B(E 2)$ values, and quadrupole moments in the negativeparity yrast states of ${ }^{127} \mathrm{La}$ and ${ }^{127} \mathrm{Ba}$.

\section{The five-dimensional quasispin formalism}

A description of configurations of neutrons and protons in a single $j$-shell is provided by the five-dimensional quasispin formalism ${ }^{8,9}$ ). It makes it possible to give the explicit $n, T$ dependence of nuclear matrix elements in the seniority scheme in terms of generalized Wigner coefficients for a five-dimensional rotation group ${ }^{10}$ ). The classification scheme is based on two parallel group chains starting with the direct product of the quasi-spin group and the symplectic group in $(2 j+1)$ dimensions, i.e. $\mathrm{R}(5) \otimes \mathrm{Sp}(2 j+1)$. The set of ten infinitesimal operators which generate $R(5)$ commute with the infinitesimal operators which generate $\mathrm{Sp}(2 j+1)$. The irreducible representations of both $\mathrm{R}(5)$ and $\mathrm{Sp}(2 j+1)$ are labelled by seniority $v$ and reduced isospin $t$. The group chain based on $\mathrm{Sp}(2 j+1)$ is that of the conventional spectroscopy of identical particle configurations, $\operatorname{Sp}(2 j+1) \supset \mathrm{R}(3)$. The group chain based on $\mathrm{R}(5)$ can be chosen to include the subgroup $\mathrm{SU}(2)$ generated by the three components of the isospin operator $T$ which commute with the number operator [generator of $U(1)]: R(5) \supset[S U(2) \otimes U(1)]$. Quasispin spectroscopy thus achieves the following aims ${ }^{10}$ ): (i) Nucleon number $n$ and isospin $T$ are associated with the lowest subgroups in one of the chains. Since they play the same role as the magnetic quantum number $M_{j}$ of ordinary angular momentum theory, the dependence on $n$ and $T$ can be factored out of any matrix element by application of a generalized Wigner-Eckart theorem and knowledge of the associated generalized $R(5)$ Wigner coefficients. (ii) The highest symmetry in the classification scheme is as simple as possible. The group $R(5)$ which is the starting point of one of the two parallel group chains is a simple group of rank 2 for which explicit properties including the needed Wigner coefficients can be worked out in detail. (iii) The starting point of that branch of the group chain containing the isospin and number 
operators is always $\mathrm{R}(5)$ so that the same symmetry group serves for all $j$ as well as for mixed configurations.

It should be pointed out that although the application of the quasi-spin formalism is straightforward in principle, it is complicated in practice by the fact that the group chain $\mathrm{R}(5) \supset \mathrm{SU}(2) \otimes \mathrm{U}(1)$ is not a canonical one corresponding to a mathematically natural group decomposition. The scheme in which the physically relevant labels $n, T$ and $M_{T}$ are good quantum numbers does not completely specify the states of the irreducible representations of $R(5)$ without the introduction of a fourth operator which commutes with $\boldsymbol{T}^{2}, T_{0}$ and the number operator. Such an operator has the disadvantage that its eigenvalues are not related to the irreducible representation labels of a subgroup of the decomposition. However, a practical resolution of this problem has been achieved ${ }^{11}$ ) for irreducible representations with reduced isospin $t \leqslant \frac{3}{2}$. Although the quasispin technique is tailor-made for the study of the general $n, T$ dependence of physical quantities for a series of nuclei, its applications in this regard are somewhat limited by the fact that it is tied closely to the seniority scheme and seniority is in general not a good quantum number in nuclei where both neutrons and protons are filling the same shells. In an isolated high $-j$ intruder orbit the effects of seniority breaking forces are inhibited, and the indications are that high- $j$ alignment effects in even-even nuclei can be accounted for through the first backbending region by a truncation scheme in which states with seniority $v \geqslant 4$ are neglected ${ }^{2}$ ).

Some observed $(n, T)$-dependent effects in light- and intermediate-weight nuclei may be understood at least qualitatively by a very simple application of the quasispin formalism if admixtures of high seniorities are relatively unimportant to the understanding of such effects. So-called isobaric mass formulae and Coulomb energy systematics may possibly fall into this category. In a systematic study of the energies of isobaric analogue states, for example, Jänecke ${ }^{12}$ ) found a simple $T(T+1)$ dependence for the energies of isobaric analogue states of odd-mass nuclei and a similar $T(T+1)$ dependence for the energies of even nuclei supplemented by a strong pairing effect which favors (lowers the energy of) the even- $T$ states of nuclei with $A=4 k,(k$ is an integer) and the odd- $T$ state of nuclei with $A=(4 k+2)$. Similarly, the isovector and tensor coefficients of the Coulomb energy (diagonal matrix elements of the Coulomb interaction) show systematic $(n, T)$-dependent effects ${ }^{13}$ ). Very similar $n, T$ dependences in the key matrix elements coupling the high- $j$ intruder orbit to the normal-parity configuration in heavy deformed odd nuclei are responsible for determining the characteristics of the odd-parity yrast bands of such nuclei.

The classification schemes of conventional spectroscopy are based on groups generated by infinitesimal operators which conserve nucleon number. The quasispin groups, on the other hand, are generated by operators which include pair creation and annihilation operators. The five-dimensional quasispin group for configurations 
$j^{n}$ is generated by the ten infinitesimal operators

$$
\begin{aligned}
A^{\dagger}\left(M_{T}\right) & =\frac{1}{2} \sum_{m, m_{t}, m_{i}^{\prime}}\left\langle\frac{1}{2} m_{t} \frac{1}{2} m_{t}^{\prime} \mid 1 M_{T}\right\rangle(-1)^{j}{ }^{m} a_{j m m_{t}}^{+} a_{j-m m_{i}^{\prime}}^{\prime} \\
& =\left[a^{+} \times a^{+}\right]_{M=0}^{J=0} i_{M_{T}}\left[\frac{1}{2}\left(j+\frac{1}{2}\right)\right]^{1 / 2}, \\
A\left(M_{T}\right) & =\left(A^{+}\left(M_{T}\right)\right)^{+}, \\
T_{ \pm} & =\sum_{m} a_{j m \pm \frac{1}{2}}^{+} a_{j m \neq \frac{1}{2}}, T_{0}=\frac{1}{2} \sum_{m}\left(a_{j m_{2} \frac{1}{2}}^{+} a_{j m_{\frac{1}{2}}}-a_{j m-\frac{1}{2}}^{+} a_{j m-\frac{1}{2}}\right),\left[\frac{1}{2} N_{\mathrm{op}}-\left(j+\frac{1}{2}\right)\right],
\end{aligned}
$$

where

$$
N_{\mathrm{op}}=\sum_{m, m_{t}} a_{j m m_{t}}^{+} a_{j m m_{t}}
$$

They are built from conventional single-nucleon creation (and annihilation) operators $a_{j m m_{t}}^{+}$(and $\left.a_{j m m_{t}}\right)$. Except for a normalization factor the operators $A^{+}\left(M_{T}\right)$ $\left(A\left(M_{T}\right)\right)$ are pair creation (annihilation) operators for nucleon pairs coupled to $J=0, T=1$ and $M_{T}$. The three components of the isospin operator $T$ and the tenth operator $H_{1}=\frac{1}{2} N_{\mathrm{op}}-\left(j+\frac{1}{2}\right)$ are the generators of the subgroup [SU(2)@U(1)] of the group $\mathrm{R}(5)$. The two commuting operators of the rank 2 group are $H_{1}=$ $\frac{1}{2} N_{\text {op }}-\left(j+\frac{1}{2}\right)$ and $H_{2}=T_{0}$ so that the weights are labelled by $n$ and $M_{T}$. The irreducible representations of $R(5)$ are labelled by $\left(\omega_{1} \omega_{2}\right)$, the highest weights $\left(H_{1 \text { eigen }}, H_{2 \text { eigen }}\right)$ of the representation. For states with seniority $v$ and reduced isospin $t$, the largest eigenvalue of $H_{1}$ is $\frac{1}{2} n_{\max }-\left(j+\frac{1}{2}\right)$ with $n_{\max }=4 j+2-v$. The state with $4 j+2-v$ nucleons ( $v$ holes) has unique isospin $t$. The largest eigenvalue of $H_{2}=\hat{T}_{0}$ in this state is thus $t$. This leads to the identification of the $\mathrm{R}(5)$ quantum numbers $\left(\omega_{1} \omega_{2}\right)$

$$
\omega_{1}=j+\frac{1}{2}-\frac{1}{2} v, \quad \omega_{2}=t,
$$

so that the irreducible representations of $R(5)$ are labelled by seniority $v$ and reduced isospin $t$. A complete labelling scheme for the states of a given irreducible representation of $R(5)$ in general requires four quantum numbers. The physics dictates the choice $n, T$ and $M_{T}$ for three of these. In general these must be supplemented by a fourth label $\beta$. For states with reduced isospin $t=1$ and $t=\frac{3}{2}$, in particular, the label $\beta$ can take on at most two values for a state of a particular $n$ and $T$. In these irreducible representations a practical resolution of the labeling problem has been made in a way which gives tractable algebraic expressions for the matrix elements of the infinitesimal operators ${ }^{11}$ ). The states for a simple configuration $j^{n}$ are thus specified by

$$
\left|\{v, t\} \beta n T M_{T} ; \alpha J M_{J}\right\rangle,
$$

where the quantum numbers $\alpha, J$ and $M_{J}$ refer to the decomposition $\operatorname{Sp}(2 j+1) \supset$ $\mathrm{R}(3) \supset \mathrm{R}(2)$. The label $\alpha$ is needed in those cases where the $v$ nucleons free of 
$J=0$ coupled pairs can be coupled to total $J$ in more than one independent way. The quantum numbers $\beta n T M_{T}$ refer to the $\mathrm{R}(5)$ branch of the group decomposition.

All physical operators can be classified according to their irreducible tensor character with respect to rotations and with respect to their five-dimensional quasispin symmetry. An R(5) irreducible tensor operator can be denoted by $T_{\beta H_{1} T M_{T}}^{\left(\omega_{1} \omega_{2}\right)}$. It should be pointed out, that the operators of interest in shell-model applications belong to the simple irreducible representations $\left(\omega_{1} \omega_{2}\right)=\left(\frac{1}{2} \frac{1}{2}\right),(10),(11),(20),(22)$ for which the label $\beta$ is unnecessary. The $R(5)$ irreducible tensor operators have the standard definitions [see e.g. eq. (7) of ref. $\left.{ }^{10}\right)$ ]. In a weak-coupled basis, a state with a number $n_{\mathrm{A}}$ of abnormal-parity nucleons in a state of good $J_{\mathrm{A}}$ and $T_{\mathrm{A}}$ coupled to a similar state with $n_{\mathrm{N}}$ normal parity nucleons, with $n_{\text {tot }}=n_{\mathrm{N}}+n_{\mathrm{A}}$, is represented by

$$
\left|n_{\mathrm{tot}} J T\right\rangle=\left[\left|n_{\mathrm{N}} J_{\mathrm{N}} T_{\mathrm{N}}\right\rangle\left|n_{\mathrm{A}} J_{\mathrm{A}} T_{\mathrm{A}}\right\rangle\right]_{J T} .
$$

A general two-body interaction leads to the following operators acting in the A-subspace. The 2-body term made up of pure A-space operators can be expressed in terms of $R(5)$ infinitesimal operators and $R(5)$ irreducible tensors of ranks $\left(\omega_{1} \omega_{2}\right)=(22)$ and $(20)$. The $\mathrm{N}$ - to A-space coupling terms include two types of A-space operators, the multipole operators, and pair-scattering operators. The multipole unit operators are defined by

$$
\begin{aligned}
{\left[a_{j}^{+} \times a_{j}\right]_{q \mu}^{k \tau}=} & \sum_{m_{1}\left(m_{2}\right)} \sum_{m_{t_{1}}\left(m_{t_{2}}\right)}\left\langle j m_{1} j m_{2} \mid k q\right\rangle\left\langle\frac{1}{2} m_{t_{1}} \frac{1}{2} m_{t_{2}} \mid \tau \mu\right\rangle \\
& \times a_{j m_{1} m_{t_{1}}}^{+} a_{j,-m_{2},-m_{t_{2}}}(-1)^{j-m_{2}+\frac{1}{2}-m_{t_{2}}} .
\end{aligned}
$$

These are spherical tensors of rank $k$, isospin rank $\tau$. For even multipoles, $k=$ even, they are $R(5)$ tensors of rank $\left(\omega_{1} \omega_{2}\right)=(11)$. For $k=$ odd they have $R(5)$ rank (10) for $\tau=1$ and $(00)$ for $\tau=0$. The pair scattering operators

$$
\left[a_{j}^{+} \times a_{j}^{+}\right]_{m \mu}^{k \tau}, \quad\left[a_{j} \times a_{j}\right]_{m \mu}^{k \tau}
$$

are coupled as in eq. (5). For $k=$ even, $\tau=1$ they have $\mathrm{R}(5)$ rank (11), whereas for $k=$ odd $\tau=0$ they have $\mathrm{R}(5)$ rank (10). (The irreducible representation (11) is the 10-dimensional regular representation of the infinitesimal operators; whereas (10) is the 5-dimensional vector representation.)

The matrix elements of a component of an $R(5)$ irreducible tensor operator can be given through a generalized Wigner-Eckart theorem:

$$
\begin{aligned}
& \left\langle\left(\omega_{1} \omega_{2}\right) \beta H_{1} T M_{T}\left|T_{\beta^{\prime \prime} H_{1}^{\prime \prime} T^{\prime \prime} M_{T}^{\prime \prime}}^{\left(\omega_{1}^{\prime \prime}\right)}\right|\left(\omega_{1}^{\prime} \omega_{2}^{\prime}\right) \beta^{\prime} H_{1}^{\prime} T^{\prime} M_{T}^{\prime}\right\rangle \\
& =\sum_{\rho}\left\langle\left(\omega_{1}^{\prime} \omega_{2}^{\prime}\right) \beta^{\prime} H_{1}^{\prime} T^{\prime} M_{T}^{\prime} ;\left(\omega_{1}^{\prime \prime} \omega_{2}^{\prime \prime}\right) \beta^{\prime \prime} H_{1}^{\prime \prime} T^{\prime \prime} M_{T}^{\prime \prime} \mid\left(\omega_{1} \omega_{2}\right) \beta H_{1} T M_{T}\right\rangle_{\rho} \\
& \quad \times\left\langle\left(\omega_{1} \omega_{2}\right)\left\|T^{\left(\omega_{1}^{\prime \prime} \omega_{2}^{\prime \prime}\right)}\right\|\left(\omega_{1}^{\prime} \omega_{2}^{\prime}\right)\right\rangle_{\rho},
\end{aligned}
$$

where the reduced or double-barred matrix elements are independent of quantum 
numbers of type $\beta, H_{1}, T$ and $M_{T}$. The dependence on these quantum numbers is carried by the first factor, an R(5) Wigner coefficient. The R(5) Wigner coefficients are the elements of the matrix which reduces the Kronecker product $\left(\omega_{1}^{\prime} \omega_{2}^{\prime}\right) \times$ $\left(\omega_{1}^{\prime \prime} \omega_{2}^{\prime \prime}\right)$ of two irreducible representations of $R(5)$. Since the isospin group is a subgroup of $R(5)$, the $R(5)$ Wigner coefficients can be factored into an ordinary isospin angular momentum Wigner coefficient and a reduced $R(5) / R(3)$ coefficient or isoscalar factor, to be denoted by a double bar:

$$
\begin{aligned}
\left\langle\left(\omega_{1}^{\prime} \omega_{2}^{\prime}\right) \beta^{\prime} H_{1}^{\prime} T^{\prime} M_{T}^{\prime} ;\left(\omega_{1}^{\prime \prime} \omega_{2}^{\prime \prime}\right) \beta^{\prime \prime} H_{1}^{\prime \prime} T^{\prime \prime} M_{T}^{\prime \prime} \mid\left(\omega_{1} \omega_{2}\right) \beta H_{1} T M_{T}\right\rangle_{\rho} \\
=\left\langle T^{\prime} M_{T}^{\prime} T^{\prime \prime} M_{T}^{\prime \prime} \mid T M_{T}\right\rangle \\
\quad \times\left\langle\left(\omega_{1}^{\prime} \omega_{2}^{\prime}\right) \beta^{\prime} H_{1}^{\prime} T^{\prime} ;\left(\omega_{1}^{\prime \prime} \omega_{2}^{\prime \prime}\right) \beta^{\prime \prime} H_{1}^{\prime \prime} T^{\prime \prime} \mid\left(\omega_{1} \omega_{2}\right) \beta H_{1} T\right\rangle_{\rho},
\end{aligned}
$$

where the double-barred coefficient, (the " $R(5)$ Wigner coefficient"), is completely independent of the quantum numbers $M_{T}$.

Tables of most of the needed $R(5)$ Wigner coefficients have been given in refs. $\left.{ }^{9-11}\right)$. The reduced matrix elements $\left\langle\left(\omega_{1} \omega_{2}\right)\left\|T^{\left(\omega_{1}^{\prime \prime} \omega_{2}^{\prime \prime}\right)}\right\|\left(\omega_{1}^{\prime} \omega_{2}^{\prime}\right)\right\rangle_{\rho}$ can then be evaluated from the calculation of the full matrix elements in a few very simple cases, e.g. between states with $n=v$. In the general case, the Wigner-Eckart theorem of eq. (7) is complicated by a sum over the multiplicity label $\rho$. The multiplicity label $\rho$ is needed in all those cases where the Kronecker product $\left(\omega_{1}^{\prime} \omega_{2}^{\prime}\right) \times\left(\omega_{1}^{\prime \prime} \omega_{2}^{\prime \prime}\right)$ contains the representation $\left(\omega_{1} \omega_{2}\right)$ with a $d$-fold multiplicity, with $d>1$. (In this case $\rho=$ $1,2, \ldots, d$.) In many cases of practical interest $d=1$, and the $\rho$-sum of eq. (7) collapses to a single term. A more challenging example is given by the matrix elements of an $R(5)$ tensor of rank (11) between states with $v=2, t=1,\left(\omega_{1} \omega_{2}\right)=$ $\left(\omega_{1} 1\right)$. In this case $d=2$. The R(5) Wigner coefficients with $\rho=1$, and $\rho-2$ are known ${ }^{10,11}$ ); but the evaluation of the $R(5)$-reduced matrix elements involves the specific construction of two states, e.g. a state with $n=v=2$, and a second state with $n=4$. Once the $\mathrm{R}(5)$-reduced matrix elements have been evaluated, the Wigner-Eckart theorem and the tabulations of reduced $R(5)$ Wigner coefficients $^{9-11}$ ) can be used to give the $(n, T)$-dependent matrix element. It will be convenient to give the results in terms of conventional angular momentum $(J)$ and isospin $(T)$ reduced matrix elements, e.g.

$$
\begin{aligned}
&\left\langle\left\{v^{\prime} t^{\prime}\right\} \beta^{\prime} n^{\prime} T^{\prime} M_{T}^{\prime} ; \alpha^{\prime} J^{\prime} M_{J}^{\prime}\left|\left[a^{+} \times a\right]_{q \mu}^{k \tau}\right|\{v t\} \beta n T M_{T} ; \alpha J M_{J}\right\rangle \\
&=\left\langle\left\langle J M_{J} k q \mid J^{\prime} M_{J}^{\prime}\right\rangle\left\langle T M_{T} \tau \mu \mid T^{\prime} M_{T}^{\prime}\right\rangle /\left[\left(2 J^{\prime}+1\right)\left(2 T^{\prime}+1\right)\right]^{1 / 2}\right. \\
& \times\left\langle\left\{v^{\prime} t^{\prime}\right\} \beta^{\prime} n^{\prime} T^{\prime} ; \alpha^{\prime} J^{\prime}\left\|\left[a^{+} \times a\right]^{k \tau}\right\|\{v t\} \beta n T ; \alpha J\right\rangle .
\end{aligned}
$$

The reduced matrix elements of greatest relevance for yrast band spectroscopy are given in the appendix. For the reasons outlined above these are limited to states with $v=0,1$, and 2. States with $v=2$ are further restricted to those with reduced isospin $t=1$, since these are the states primarily responsible for the high- $j$ alignment effects through the first backbending region of the yrast bands of even nuclei. The 
companion states with $v=2$ and $t=0$ are generally restricted to states with $T>M_{T}$ and thus lie too high in energy to play a significant role. E.g. in a nucleus in which the abnormal-parity configuration $\left(h_{11 / 2}\right)^{10}$ with $T=M_{T}=3$ is dominant in the $J=0$ ground state, the represention with $\{v, t\}=\{2,1\}$ contains two states with $T=3$; $\beta=1$ and 2 . Since the "quantum number" $\beta$ was chosen largely for practical reasons of calculational simplicity both states must be included. On the other hand, the representation with $\{v, t\}=\{20\}$ has no states with $T=3$ for $n=10$. The $T=4$ states with $\{v, t\}=\{20\}$ can be expected to lie at an energy of $\sim 2 \mathrm{MeV}$ above those with $\{v, t\}=\{21\}$ and $T=3$ and would therefore lead to negligible admixtures to the low-lying states in such a nucleus.

\section{Calculations for ${ }^{126,127} \mathrm{Ba}$ and ${ }^{127} \mathrm{La}$}

The microscopic hamiltonian to be diagonalized is chosen to have the form

$$
H=H_{\text {s.p. }}+H_{2-\text { body }}+Q_{\mathrm{A}} \cdot Q_{\mathrm{N}} \text {. }
$$

The single-particle energies of the $g_{7 / 2}, d_{5 / 2}, d_{3 / 2}, s_{1 / 2}$ and $h_{11 / 2}$ levels are given the values $0.0,0.4,2.8,3.2$ and $1.5 \mathrm{MeV}$, respectively. The two-body interaction is chosen to be a surface delta interaction (active in both the normal- and abnormalparity spaces) with strength coefficient $G=0.29 \mathrm{MeV}$ [see ref. ${ }^{2}$ )]. The $Q \cdot Q$ multipole interaction between normal- and abnormal-parity spaces had a strength equal to $0.06 \mathrm{MeV}$ which is 0.8 of the self-consistent value $240 A^{-5 / 3}$ for this region. The hamiltonian was then decomposed into its pseudo-SU(3) irreducible tensor components and matrix elements were then calculated.

A detailed analysis of the backbending phenomena in ${ }^{126} \mathrm{Ba}$ has already appeared ${ }^{2}$ ). The partition of the normal-parity part of the shell-model space which dominated the low-energy yrast states was found to be [444222]. The leading pseudo-SU(3) irrep of this partition is $(24,0)$. In this work we restrict our basis to that irrep only. The abnormal-parity subspace configurations which belong to this partition are the configurations $\left(\mathrm{h}_{11 / 2}\right)^{8} T=4,\left(\mathrm{~h}_{11 / 2}\right)^{9} T=\frac{9}{2}$, and the $\left(\mathrm{h}_{11 / 2}\right)^{9} T=\frac{7}{2}$ for ${ }^{126} \mathrm{Ba},{ }^{127} \mathrm{Ba}$ and ${ }^{127} \mathrm{La}$, respectively. For the negative-parity states of ${ }^{127} \mathrm{Ba}$ and ${ }^{127} \mathrm{La}$ we restrict the configuration to $v=1$, while $v=0$ and $v=2$ have been included in the ${ }^{126} \mathrm{Ba}$ basis.

The result of diagonalizing $\mathrm{H}$ in the three cases is shown in fig. 1 . For ${ }^{126} \mathrm{Ba}$ the yrast wave functions were dominated by $v=0$ configurations in the $h_{11 / 2}$ space. It should be recalled that in the normal-parity space each pseudo-SU(3) basis state represents a large mixing of shell-model seniority.

Previous ${ }^{7}$ ) analysis of the five-dimensional quasi spin matrix elements from table 6 implied that a prolate deformed even-even parent (as is ${ }^{126} \mathrm{Ba}$ ) should give rise to a negative-parity excitation spectrum in the neighboring odd nucleus which is a $\Delta I=2(\Delta I=1)$ rotational sequence if a proton (neutron) is added, i.e. ${ }^{127} \mathrm{La}$ (or ${ }^{127} \mathrm{Ba}$ ). Fig. 1 clearly upholds this analysis. 


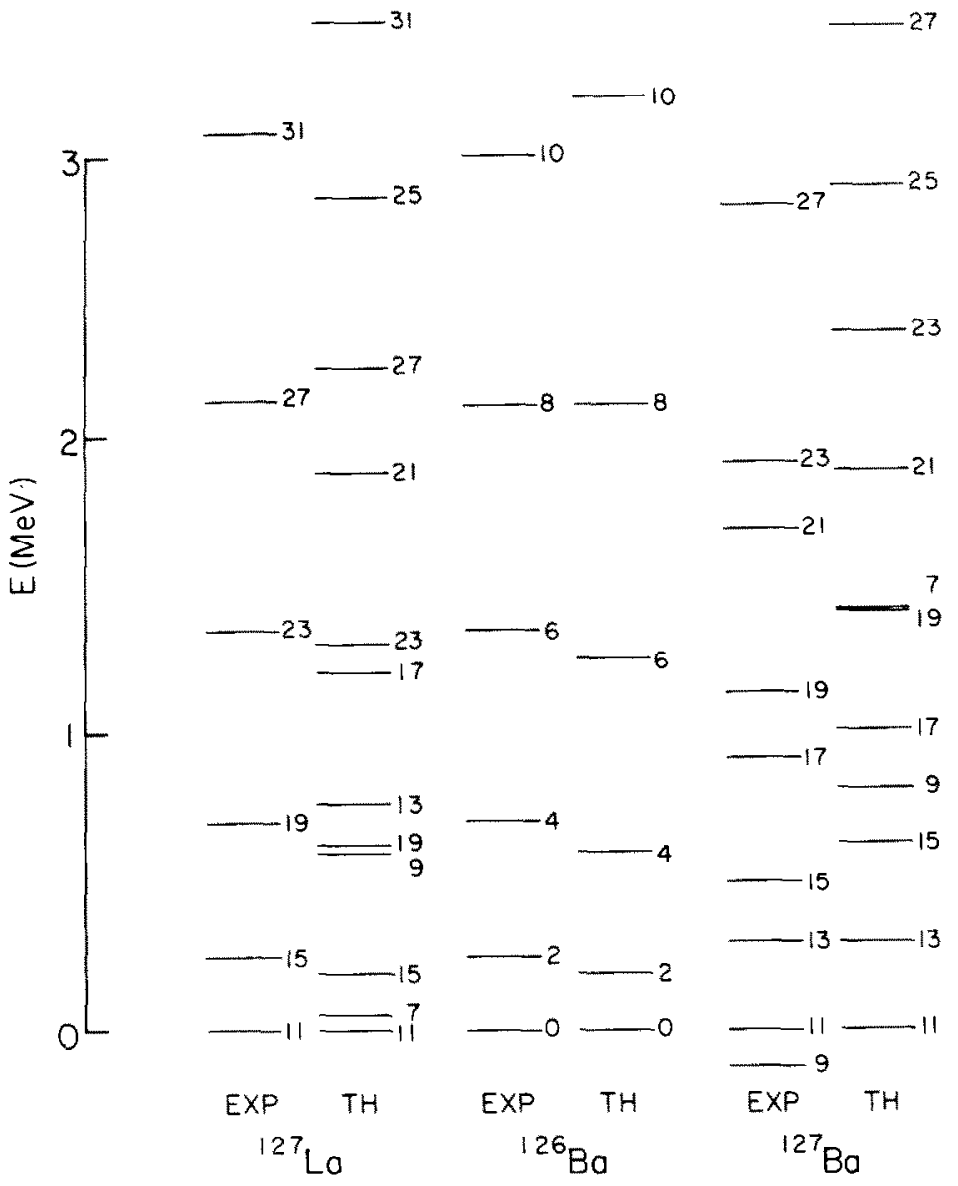

Fig. 1. Theoretical and experimental ${ }^{14,15}$ ) excitation energies for ${ }^{127} \mathrm{La},{ }^{126} \mathrm{Ba}$ and ${ }^{127} \mathrm{Ba}$. For ${ }^{127} \mathrm{Ba}$ and ${ }^{127} \mathrm{La}$ the states are labelled by $2 I$.

If an SU(3) tensor decomposition is made of the microscopic quadrupole operator, we may calculate transition matrix elements with our wave functions. Assigning a charge of $0.5 e(1.5 e)$ for the neutrons (protons), i.e. an effective charge of $0.5 e$, we have calculated quadrupole moments and $B(\mathrm{E} 2)$ 's.

In fig. 2 we observe that the predicted $Q$-moments of ${ }^{127} \mathrm{La}$ follow its even-even parent closely. The favored states of ${ }^{127} \mathrm{La}$ (i.e. $I=\frac{11}{2}+2 n, n=0,1,2 \ldots$ ) are slightly more deformed than the unfavored states $\left(I=\frac{11}{2}+2 n+1, n=0,1,2, \ldots\right) .{ }^{127} \mathrm{Ba}$ shows interesting behavior. For $I<\frac{17}{2}$, the quadrupole moments are positive. As the angular momentum increases, the quadrupole moments gradually approach the ${ }^{126} \mathrm{Ba}$ results.

Comparing $B(\mathrm{E} 2 ; I \rightarrow I-2)$ in the lower half of fig. 3 we find similar behavior, i.e. ${ }^{127} \mathrm{La}$ follows ${ }^{126} \mathrm{Ba}$ whereas ${ }^{127} \mathrm{Ba}$ does not. Note that the $B\left(\mathrm{E} 2 ; \frac{14-}{2} \rightarrow\right.$ $\left.\frac{15}{2}^{-}\right) / B\left(\mathrm{E} 2 ; \frac{15}{2}^{-} \rightarrow \frac{11}{2}^{-}\right)$ratio is about unity in ${ }^{127} \mathrm{Ba}$, whereas in ${ }^{126} \mathrm{Ba}$, 


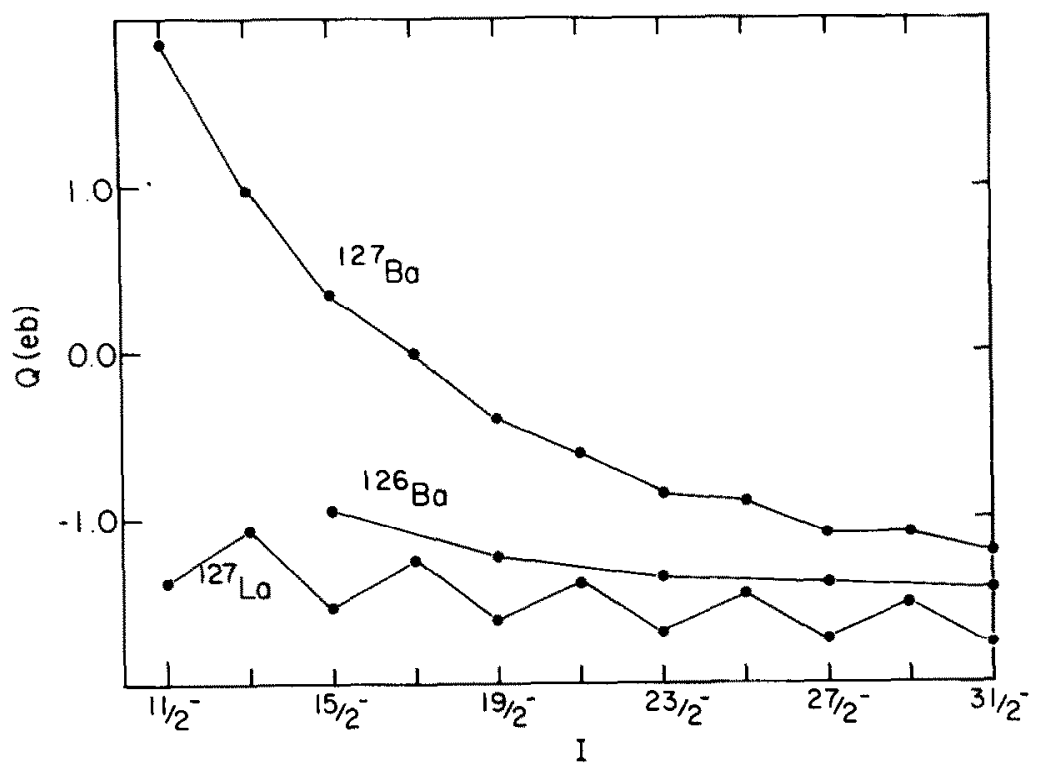

Fig. 2. Theoretical quadrupole moment predictions for ${ }^{127} \mathrm{La},{ }^{126} \mathrm{Ba}$ and ${ }^{127} \mathrm{Ba}$.

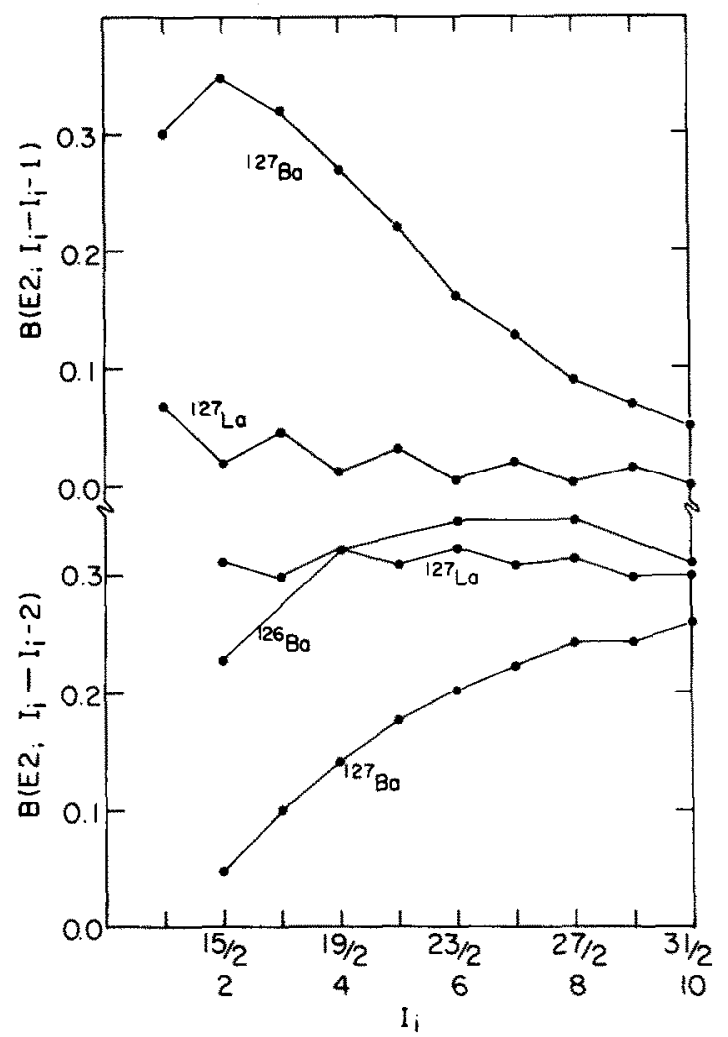

Fig. 3. Theoretical $B\left(E 2 ; I_{\mathrm{i}} \rightarrow I_{\mathrm{i}}-1\right)$ and $B\left(\mathrm{E} 2 ; I_{\mathrm{i}} \rightarrow I_{\mathrm{i}}-2\right)$, integer $I_{\mathrm{i}}$ refers to the ${ }^{126} \mathrm{Ba}$ case, while half-integer refers to the ${ }^{127} \mathrm{Ba}$ and ${ }^{127} \mathrm{La}$ cases. 
$B(\mathrm{E} 2 ; 4 \rightarrow 2) / B(\mathrm{E} 2 ; 2 \rightarrow 0)$ is around 1.4 . Thus we see that the ${ }^{127} \mathrm{La}$ seniority-one results are not quite the same as those from weak-coupling the odd proton to the ${ }^{126} \mathrm{Ba}$ core. Also in fig. 3 we see that it is the states $I$ and $I-2(I-1)$ which are strongly connected by the E2 operator in ${ }^{127} \mathrm{La}\left({ }^{127} \mathrm{Ba}\right)$.

One notes in fig. 1 that the energies of the abnormal-parity states of ${ }^{127} \mathrm{Ba}$ are not reproduced well by the calculation. It is thus clear that the configurations based on pure $v=1 \mathrm{~h}_{11 / 2}$ truncations are not enough to describe the negative-parity states of ${ }^{127} \mathrm{Ba}$. Higher-seniority mixing is required. For ${ }^{127} \mathrm{La}$, on the other hand, the energies seem well described by just the $v=1$ basis. This and the results of figs. 2 and 3 point out an essential difference between ${ }^{127} \mathrm{Ba}$ and ${ }^{127} \mathrm{La}$. Namely, the odd nucleon tends to destroy the even-even parent structure of ${ }^{126} \mathrm{Ba}$ in the case of ${ }^{127} \mathrm{Ba}$ whereas this does not occur in ${ }^{127} \mathrm{La}$. In ${ }^{127} \mathrm{La}$, the odd proton may take an orientation which reinforces the deformation of the ${ }^{127} \mathrm{Ba}$ parent. However, in ${ }^{127} \mathrm{Ba}$, the odd neutron is prevented from doing so by the Pauli Principle. Since the neutron is not paired off it generates its own quadrupole field which overlaps most strongly with a normal-parity structure which was not the dominant structure in the eveneven parent. As pairs of particles are broken to generate higher angular momentum states ( $I$ increasing) the ability of the odd nucleon to polarize the other nucleons is considerably reduced. Therefore as $I$ increases, the ${ }^{127} \mathrm{Ba}$ results gradually tend toward those for ${ }^{126} \mathrm{Ba}$ and ${ }^{127} \mathrm{La}$.

\section{Discussion}

In conclusion, we have shown that within the weak coupled pseudo-SU(3) scheme, we may describe the yrast states of even and odd nuclei neighbors, at least qualitatively, in a microscopic shell-model framework. The formalism of the fivedimensional quasispin description of a single $j$-shell has been outlined and many of the most important matrix elements of one- and two-body operators have been tabulated. This formalism, of course, may be of use in alternate microscopic shellmodel analyses.

Finally it was found that when applied to the Ba region, higher-seniority configurations are needed to describe the low-energy abnormal-parity states of odd nuclei for nuclei whose spectra have the character of $\Delta I=1$ rotational sequences. The calculation of such matrix elements we reserve to future work.

\section{Appendix}

Tables 1-7 give the conventional reduced (with respect to angular momentum and isospin) matrix elements of various operators of physical interest. The wave functions are labelcd as in (3), except that $\alpha$ is not needed so it is omitted. Primed (unprimed) quantum numbers refer to the bra (ket) state in the matrix element. A circumflex above a quantum number denotes the square root of twice the 
number plus one, e.g. $\hat{J} \equiv \sqrt{2 J+1}$. In every table the letters $A$ through $G$ have the following definitions:

$$
\begin{aligned}
& A \equiv j-\frac{1}{2}, \quad B \equiv j+\frac{1}{2}, \quad C \equiv T+1, \quad D \equiv \frac{1}{2} n, \quad E \equiv j+\frac{3}{2}, \\
& F(a, b) \equiv(b+1)(E+b)(B-b)+b(a-D)^{2}, \quad G \equiv \hat{T} \hat{\jmath} \hat{J}_{0} \hat{J}^{\prime} W\left(J_{0} j J j ; j J^{\prime}\right) .
\end{aligned}
$$

$W$ is a Racah coefficient. The RME's satisfy the following conjugation property

$$
\left\langle J^{\prime} T^{\prime}\|\theta\| J T\right\rangle=(-)^{J^{\prime}-J+T^{\prime}-T}\left\langle J T\left\|\theta^{\dagger}\right\| J^{\prime} T^{\prime}\right) .
$$

TABLE 1

One-body $R M E=\langle\{v, t\} \beta n T$; $\left.J\left\|\left[a^{\dagger} a\right]^{J_{0}=0, x_{0}}\right\|\{v, t\} \beta n T ; J\right\rangle$ for any $\{v, t\} \beta$

\begin{tabular}{cc}
\hline$T_{0}$ & $\mathrm{RME}$ \\
\hline 1 & $\hat{T} \hat{I} \sqrt{T C / B}$ \\
0 & $D \hat{T} \hat{\jmath} / \sqrt{B}$ \\
\hline
\end{tabular}

TABLE 2

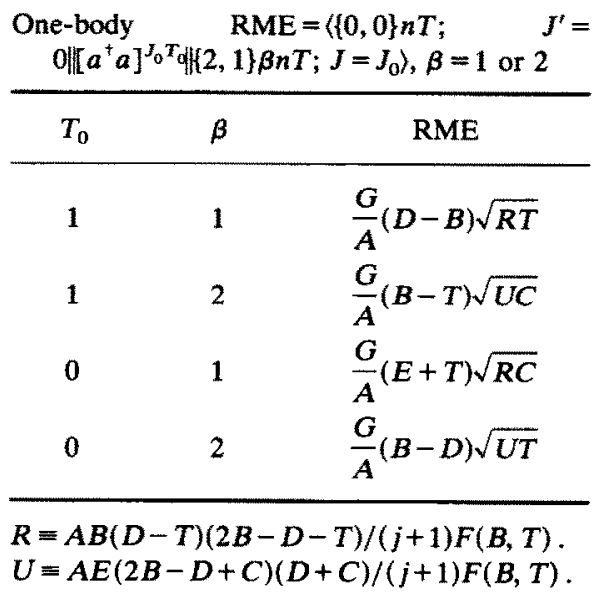

TABLE 3

One-body RME $=\left\{\{2,1\} \beta^{\prime} n T ; J^{\prime} \|\left[a^{\dagger} a\right]^{J_{0}=\text { odd, }}\right.$ o\| $\left.[2,1\} \beta n T ; J\right\rangle$

\begin{tabular}{cccl}
\hline$T_{0}$ & $\beta^{\prime}$ & $\beta$ & RME \\
\hline 0 & 2 & 2 & $\sqrt{2} G \delta_{\beta^{\prime} 2} \delta_{\beta 2}$ \\
1 & 2 & 2 & $\frac{E G(B-D) \hat{T}^{2}}{F(B, T)} \sqrt{\frac{2 C}{T}}$ \\
1 & 1 & 2 & $\frac{2 G C}{\hat{j} F(B, T)}[E(D-T)(2 B-D-T)(D+C)(2 B+C-D)\}^{1 / 2}$ \\
1 & 1 & 1 & $\frac{G(B-D)}{B F(B, T)}\left[2 B E C+C^{2}-(B-D)^{2}\right] \sqrt{\frac{2 T}{C}}$ \\
\hline
\end{tabular}


TABLE 4

One-body RME $=\left\langle\{2,1\} \beta^{\prime} n T ; J^{\prime} \mid\left[a^{\dagger} a\right]^{J_{0}=\text { even, }} T_{0} \|\{2,1\} \beta n T ; J\right\rangle$

\begin{tabular}{cccc}
\hline$T_{0}$ & $\beta^{\prime}$ & $\beta$ & $R M E$ \\
\hline 0 & 2 & 2 & $\frac{S(D-B)}{\sqrt{B E}}\left[(E+1) F(B, T)-C \hat{T}^{2} B E\right]$ \\
0 & 2 & 1 & $S C\{T C(2 B-T-D)(D-T)(D+C)(2 B+C-D)\}^{1 / 2}$ \\
0 & 1 & 1 & $\frac{S(D-B)}{\sqrt{B E}}\left[(B+1) F(B, T)+C \hat{T}^{2} B E\right]$ \\
1 & 2 & 2 & $\frac{S C}{\sqrt{T C B E}}\left[T(E+1) F(B, T)-B E\left((E+T)(B-T)+2 T(D-B)^{2}\right)\right]$ \\
1 & 2 & 1 & $S(D-B) T\{(D-T)(2 B-D-T)(D+C)(2 B-D+C)\}^{1 / 2}$ \\
1 & 1 & 1 & $\frac{S T E}{\sqrt{B E T C}}\left[C F(B, T)+(D-B)^{2} \hat{T}^{2} B\right]$
\end{tabular}

$S \equiv-G \hat{j} /(A-1) F(B, T) \sqrt{E}$.

TABLE 5

Pair-annihilation RME $=\left\langle\left\{v^{\prime}, t^{\prime}\right\} \beta^{\prime} n-2 T^{\prime} ; J^{\prime} \mid[a a]^{J_{0}, T_{0}=1} \|\{v, t\} \beta n T ; J\right\rangle$

\begin{tabular}{|c|c|c|c|c|}
\hline$v^{\prime}, t^{\prime}, \beta^{\prime}$ & $T$ & $v, t, \beta$ & $J_{0}$ & RME \\
\hline 0,0 & $T^{\prime}+1$ & 0,0 & 0 & $-\left\{\frac{2 T(D+C)(2 E-D-T)}{2 j+1}\right\}^{1 / 2}$ \\
\hline 0,0 & $T^{\prime}-1$ & 0,0 & 0 & $\left\{\frac{2 C(D-T)(2 E-D+C)}{2 j+1}\right\}^{1 / 2}$ \\
\hline 0,0 & $T^{\prime}+1$ & $2,1,2$ & $J$ & $-\hat{J}[B C-T(C+D-B)]\left\{\frac{E(2 B-D+C)(2 E-D-T)}{A B(2 B+1) F(B, T)}\right\}^{1 / 2}$ \\
\hline 0,0, & $T^{\prime}-1$ & $2,1,2$ & $J$ & $-\hat{J}\left\{\frac{E C T(D-T)(2 B+C-D)(D+C)(2 E-D+C)}{A B(2 B+1) F(B, T)}\right\}^{1 / 2}$ \\
\hline 0,0 , & $T^{\prime}+1$ & $2,1,1$ & $J$ & $-\hat{J}\left\{\frac{T C(D+C)(D-T)(2 E-D-T)(2 B-D-T)}{A(2 B+1) F(B, T)}\right\}^{1 / 2}$ \\
\hline 0,0 & $T^{\prime}-1$ & $2,1,1$ & $J$ & $\hat{J}[C E+T(T+E-D)]\left\{\frac{(2 B-D-T)(2 E+C-D)}{A(2 B+1) F(B, T)}\right\}^{1 / 2}$ \\
\hline $2,1,2$ & $T^{\prime}+1$ & 0,0 & $J^{\prime}$ & $\hat{J}^{\prime}\left\{\frac{E T T^{\prime}(2 E-D-T)(D+T-1)(2 B+C-D)(D+C)}{A B(2 B+1) F\left(E, T^{\prime}\right)}\right\}^{1 / 2}$ \\
\hline $2,1,2$ & $T^{\prime}-1$ & 0,0 & $J^{\prime}$ & $\begin{array}{c}\hat{J}^{\prime}\left[B(T+2)-T^{\prime}(T+2+E-D)\right] \\
\quad \times\left\{\frac{E(D+C)(D-T)}{A B(2 B+1) F\left(E, T^{\prime}\right)}\right\}^{1 / 2}\end{array}$ \\
\hline $2,1,1$ & $T^{\prime}+1$ & 0,0 & $J^{\prime}$ & $\hat{J}^{\prime}\left[T E+T^{\prime}(D+T-E)\right]\left\{\frac{(D-T)(D+C)}{A B F\left(E, T^{\prime}\right)}\right\}^{1 / 2}$ \\
\hline
\end{tabular}


TABLE 5 (contd.)

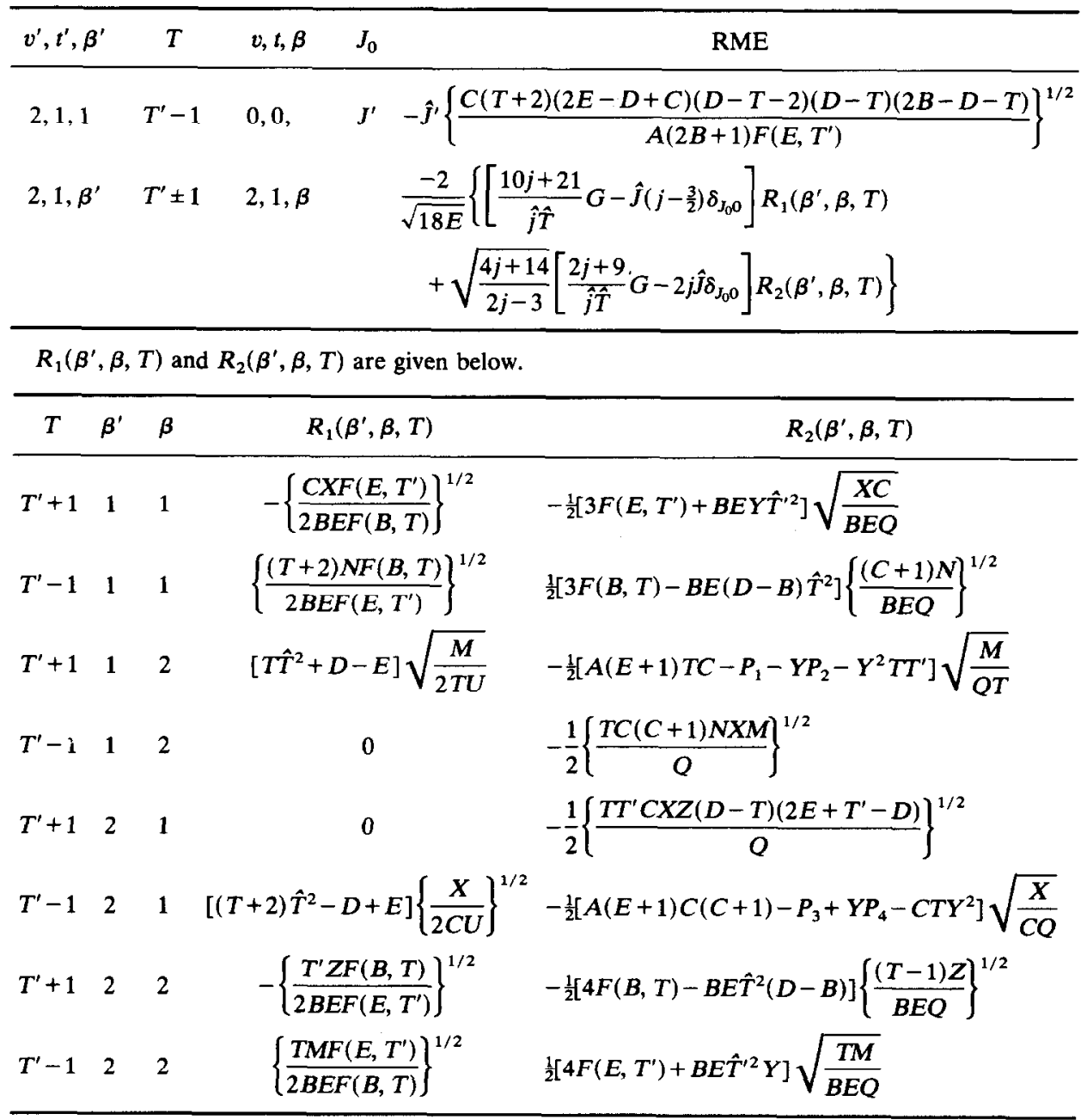

$S \equiv(2 E+C-D)(D-T)$,

$U \equiv F(B, T) F\left(E, T^{\prime}\right)$,

$X \equiv(2 B-D-T)(D+C)$,

$Q \equiv(2 j-E)(E+2) U$,

$Z \equiv(2 E-D-T)\left(D+T^{\prime}\right)$,

$Y \equiv D-E$,

$M \equiv(2 B+C-D)(D-T)$,

$N=(2 E+C-D)(D-C-1)$,

$P_{1} \equiv T^{4}+2 T^{3}+4 T^{2}+T$,

$P_{2} \equiv 2 T^{3}+T^{2}-2 T+3$,

$P_{3} \equiv T^{4}+4 T^{3}+10 T^{2}+14 T+4$,

$P_{4} \equiv 2 T^{3}+5 T^{2}+4 C$. 
TABLE 6

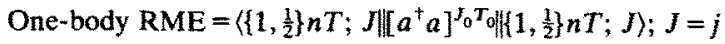

\begin{tabular}{ccc}
\hline$J_{0}$ & $T_{0}$ & $\mathrm{RME}$ \\
\hline odd & 0 & $K$ \\
odd & 1 & $K \sqrt{T C}\left[\frac{\left.B-D+(-)^{D-T} E \hat{T}^{2}\right]}{2 T C(j+1)}\right]$ \\
even $\neq 0$ & 0 & $\frac{K}{L}\left[4 E(B-D)-\frac{B\left(20(D-B) E+(-)^{D-T} \hat{T}^{2} L\right)}{(j+1)(2 j-1)}\right]$ \\
even $\neq 0$ & 1 & $\frac{-2 K}{L} \sqrt{T C}\left[2 E+B \frac{\left(20 E T C-L\left(E-(-)^{D-T}(D-B) \hat{T}^{2}\right)\right)}{4 C A T(j+1)}\right]$ \\
\hline$K \equiv \sqrt{\frac{1}{2} \hat{T} \hat{J}_{0},}$ & $L \equiv 4 j^{2}+12 j+3$. &
\end{tabular}

TABLE 7

Pair-annihilation RME $=\left\{\left\{1, \frac{1}{2}\right\} n-2 T^{\prime} ; J=j \|[a a]^{J_{0}} T_{0 \|}\left(1, \frac{1}{2}\right\} n T ; J=j\right)=\sqrt{\frac{1}{2}} \hat{J}_{0} \hat{T}^{\prime} M X\left(J_{0}\right)$

\begin{tabular}{|c|c|c|c|c|c|c|}
\hline$J_{0}$ & $T_{0}$ & $T$ & $\frac{1}{2} n-T^{\prime}$ & $M$ & $X\left(J_{0} \neq 0\right)$ & $X\left(J_{0}=0\right)$ \\
\hline even & 1 & $T^{\prime}+1$ & even & $\hat{T}\left\{\frac{(2 E-D-T)(D+C)}{T}\right\}^{1 / 2}$ & $\frac{1}{2 A(j+1)}$ & -1 \\
\hline even & 1 & $T^{\prime}-1$ & even & $\hat{T}\left\{\frac{(2 E-D+T)(D-C)}{C}\right\}^{1 / 2}$ & $-\frac{1}{A}$ & 1 \\
\hline even & 1 & $T^{*}$ & even & $\left\{\frac{(2 E-D-C)(D-T)}{C T}\right\}^{1 / 2}$ & $-\frac{2 B C+1}{2 A(j+1)}$ & 1 \\
\hline even & 1 & $T^{\prime}+1$ & odd & $\hat{T}\left\{\frac{(2 E-D-C)(D+T)}{T}\right\}^{1 / 2}$ & $\frac{1}{A}$ & -1 \\
\hline even & 1 & $T^{\prime}-1$ & odd & $\hat{T}\left\{\frac{(2 E-D+C)(D-T)}{C}\right\}^{1 / 2}$ & $\frac{-1}{2 A(j+1)}$ & 1 \\
\hline even & 1 & $T^{\prime}$ & odd & $\left\{\frac{(2 E-D+T)(C+D)}{T C}\right\}^{1 / 2}$ & $\frac{2 B T-1}{2 A(j+1)}$ & 1 \\
\hline odd & 0 & $T^{t}$ & even & $\{(D-T)(2 E-D-C)\}^{1 / 2}$ & $\frac{-1}{j+1}$ & \\
\hline odd & 0 & $T^{\prime}$ & odd & $\{(D+C)(2 E-D+T)\}^{1 / 2}$ & $\frac{-1}{j+1}$ & \\
\hline
\end{tabular}

For completeness we include table 8 in which we give the two-body matrix elements which have appeared previously ${ }^{10,11}$ ). Note that a few typographical errors which appeared in ref. ${ }^{11}$ ) have been corrected. 
TABLE 8

Two-body ME $=\left\langle\{v, t\} \beta^{\prime} n T ; J\left|\frac{1}{2} \sum_{J_{0} T_{0}} V_{J_{0} T_{0}}\left[a^{\dagger} a^{\dagger}\right]^{J_{0} T_{0}} \cdot[a a]^{J_{0} T_{0}}\right|\{v, t\} \beta n T ; J\right\rangle$, where $V_{J_{0} T_{0}}$ arc constants

if $\{v, t\}=\{2,1\}$; and $\beta^{\prime}=\beta, \beta=1,2$ :

$$
\begin{aligned}
\mathrm{ME}= & H\left(3 j V_{\mathrm{e}}+(j+1) V_{\mathrm{o}}\right)+\frac{3}{20}\left[(2 j-3) V(0)+(12 j+2) j V_{\mathrm{e}}+4 j V_{\mathrm{ew}}+4 V(J)\right] \\
& +\frac{1}{5}\left[2(j+1)^{2} V_{\mathrm{o}}-(j+1) V_{\mathrm{ow}}\right]-\frac{B E-2 T C-4 H^{2}}{6 A}\left[\frac{R}{2}-\frac{2 B X}{j}\right] \\
& +\frac{B E-5 T C+5 H^{2}}{60(j+1)}\left[2 S-\frac{Y}{j B E}\right]-\frac{Y}{15 j} \\
& +\frac{(-)^{\beta}}{3 j^{2}}\left[\frac{Y}{E}-2(B+3 T) X\right]\left[(E+T)(B-T)+H^{2}-\frac{2 H^{2} B E \hat{T}^{2}}{F(B, T)}\right] \\
& -X\left[\frac{3 B E+T C\left(j-\frac{5}{2}\right)-B H^{2}-3(-)^{\beta} F(B, T)}{3 j B}\right]
\end{aligned}
$$

if $\{v, t\}=\{2,1\} ;$ and $\beta^{\prime}=1, \beta=2:$

$$
\mathrm{ME}=\frac{-H}{3 j F(B, T)}(2 E X(B+3 T)-Y)\left\{\frac{T C\left[(E+T)^{2}-H^{2}\right]\left[(B-T)^{2}-H^{2}\right]}{B E}\right\}^{1 / 2}
$$

if $\{v, t\}=\{0,0\}$ or $\left\{1, \frac{1}{2}\right\} ; \beta^{\prime}=\beta$ :

$$
\begin{aligned}
\mathrm{ME}= & D(n-1) \frac{(6 j+5) j V_{\mathrm{e}}-2 E V(0)+2 A(j+1) V_{\mathrm{o}}}{8 A(j+1)} \\
& +\left(C T-\frac{3}{4} n\right) \frac{E j V_{\mathrm{e}}-B V(0)-A(j+1) V_{\mathrm{o}}}{2 A(j+1)}+\left[\frac{1}{2} n\right] \frac{j\left(V(0)-V_{\mathrm{e}}\right)}{A}
\end{aligned}
$$

$$
\begin{aligned}
& {\left[\frac{1}{2} n\right] \equiv \begin{cases}D, & \text { if } v=0 \\
D-\frac{1}{2}, & \text { if } v=1\end{cases} } \\
& H=D-B \text {, } \\
& R=2\left(2 j V_{\mathrm{e}}-V(0)\right) \text {, } \\
& S=j V_{\mathrm{e}}+V(0)+3(j+1) V_{\mathrm{o}}, \\
& X=\frac{2\left(2 j V_{\mathrm{e}}-V(0)\right)+2 A\left(2 j V_{\mathrm{ew}}-V(J)\right)}{(2 j-3)(2 j-5)}, \\
& Y=\frac{1}{2}\left[j V_{\mathrm{e}}+V(0)-2(j+1)\left(j V_{\mathrm{ew}}+V(J)\right)+3(j+1) V_{\mathrm{o}}-6(j+1)^{2} V_{\mathrm{ow}}\right] \text {, } \\
& V_{\mathrm{e}} \equiv \frac{\sum_{\mathrm{even} L} V_{1}(2 I+1)}{j(2 j+1)} \quad V_{\mathrm{o}} \equiv \frac{\sum_{\mathrm{oddl}} V_{I 0}(2 I+1)}{(j+1)(2 j+1)} \\
& V_{\text {ew }}=\frac{1}{j} \sum_{\text {even } i} V_{I 1}(2 I+1) W(I j j J ; j j) \text {, } \\
& V_{\mathrm{ow}} \equiv \frac{1}{(j+1)} \sum_{\text {odd } I} V_{I 0}(2 I+1) W(I j j ; j) \text {, } \\
& V(J)= \begin{cases}V_{Y, T=0}, & \text { if odd } J \\
V_{X, T=1}, & \text { if even } J .\end{cases}
\end{aligned}
$$




\section{References}

1) J.P. Draaycr, K.J. Wecks and K.T. Hecht, Nucl, Phys. A381 (1982) 1

2) J.P. Draayer, C.S. Han, K.J. Weeks and K.T. Hecht, Nucl. Phys. A365 (1981) 127; K.J. Weeks, J.P. Draayer and C.S. Han, Nucl. Phys. A371 (1981) 19

3) R.D. Ratna Raju, J.P. Draayer and K.T. Hecht, Nucl. Phys. A202 (1973) 433

4) A. Arima, M. Harvey and K. Shimizu, Phys. Lett. 30B (1969) 517;

K.T. Hecht and A. Adler, Nucl. Phys. A137 (1969) 129;

R. Arvieu, Proc. Int. School of Physics "Enrico Fermi", course XL, ed. M. Jean and R.A. Ricci, (Academic Press, New York, 1969) 631

5) J.P. Elliot, Proc. Roy. Soc. A245 (1958) 128, 562;

J.P. Elliot and M. Harvey, Proc. Roy. Soc. A272 (1963) 557

6) A. Bohr, I. Hamamoto and B.R. Mottelson, Phys. Scripta 26 (1982) 267

7) K.J. Weeks and J.P. Draayer, Nucl. Phys. A393 (1983) 69

8) K. Helmers, Nucl. Phys. 23 (1961) 594;

B.H. Flowers and S. Szpikowski, Proc. Phys. Soc. 84 (1964) 193;

J.C. Parikh, Nucl. Phys. 63 (1965) 214;

M. Ichimura, Progr. Theor. Phys. 32 (1964) 767;

J.N. Ginocchio, Nucl. Phys. 74 (1965) 321

9) K.T. Hecht, Phys. Rev. 139B (1965) 794

10) K.T. Hecht, Nucl. Phys. A102 (1967) 11

11) R.P. Hemenger, Ph.D. Univ. of Michigan (1968), unpublished;

R.P. Hemenger and K.T. Hecht, Nucl. Phys. A145 (1970) 468

12) J. Jänecke, Nucl. Phys. 73 (1965) 97

13) J. Jänecke, Isobaric spin in nuclear physics (Academic Press, New York, 1966) p. 60; Phys. Rev. 147 (1966) 735

14) C.M. Lederer and V.S. Shirley, ed., Table of isotopes, 7 th ed. (Wiley, New York, 1978)

15) J. Gizon and A. Gizon, Z. Phys. A281 (1977) 99 